\title{
Revisão sistemática das diretrizes de análise de impacto orçamentário
}

INTRODUÇÃO: As diretrizes para Análise de Impacto Orçamentário (AIO) têm a finalidade de padronizar os estudos, tornando-os úteis e compreensíveis aos gestores. Avaliar a concordância dessas diretrizes aos instrumentos disponíveis na literatura pode contribuir no conhecimento dos itens relevantes para padronização e transparência dos estudos de AIO. OBJETIVO: Revisar sistematicamente as diretrizes para a elaboração de AIO. MÉTODOS: Foram utilizadas as bases de dados MEDLINE, EMBASE, Cochrane Library, Economic Evaluation Database (NHS EED), HTA Database (via CRD) e LILACS; websites de organizações de avaliações de tecnologias em saúde e Ministérios da Saúde dos países. O critério de inclusão foi tratar-se de diretriz metodológica com recomendações para a elaboração de AIO. Dois revisores realizaram independentemente a seleção das diretrizes e extração dos dados e um terceiro revisor avaliou as discordâncias. Os instrumentos utilizados foram as recomendações de boas práticas para elaboração de AIO da Internacional Society for Pharmacoeconomic and Outcomes Research (ISPOR) e Appraisal of Guidelines for Research \& Evaluation Instrument (AGREE II). Esta revisão foi relatada de acordo com o Preferred Reporting Items for Systematic Review and Meta-Analysis Protocols (PRISMA). RESULTADOS: Foram incluídas 17 diretrizes publicadas entre 2006 a 2018, sendo sete diretrizes específicas para AIO, com concordância às recomendações da ISPOR de 30\% a 92\% e as diretrizes incluídas em documentos de avaliação econômica em saúde (AES), concordaram de 19\% a 70\%. A avaliação metodológica com AGREE II foi de 18\% (DP $\pm 12 \%$ ) no domínio rigor metodológico e $15 \%$ ( $\mathrm{DP} \pm 23 \%$ ) no domínio independência; para as diretrizes específicas para AIO, a pontuação foi $26 \%$ (DP $\pm 32 \%$ ) e as diretrizes com mais de um tipo AES, $8 \%$ (DP $\pm 13 \%$ ). CONCLUSÃO: A concordância das diretrizes para a elaboração de AIO foi maior nas diretrizes específicas para AIO. Na análise da metodologia, 15 diretrizes apresentaram baixa qualidade metodológica no domínio rigor metodológico. 